\title{
Design and application of interface circuit of coal mine equipment intelligent management system TCP/IP module
}

\author{
Xingshan $\mathrm{LI}^{1, \mathrm{a}}$, Jian $\mathrm{LU}^{2}$ \\ ${ }^{1}$ Luohe medical college, Luohe, 462000, China \\ ${ }^{2}$ Henan Polytechnic Institute, Nanyang, 473000, China \\ aemail:604141388@qq.com
} Keywords: Coal mine blasting material library, intelligentialize, SCM, NP801, serial port,
communication

\begin{abstract}
Coal mine blasting equipment library intelligent management to regulate store of blasting equipment through real-time monitoring of blasting equipment storage environment (temperature, humidity, pressure and vibration). Using fingerprint identification to open the door, by computer or mobile phone remote for authorization. The behavior of open or close door and real-time monitoring data recorded by the computer system for the record. TCP/IP module interface circuit to make the system communication more safe and reliable. Design of TCP/IP module interface circuit run normally through test.
\end{abstract}

\section{System architecture}

The intelligent management system of the explosive device database is made up of the computer access control system (lower machine)installed on the database, the database in the server and the management terminal software( management system or PC) in PC[1]. The management terminal software is set up to four kinds of users: the leader, the system administrator, the security officer, the warehouse keeper. As shown in Fig. 1.

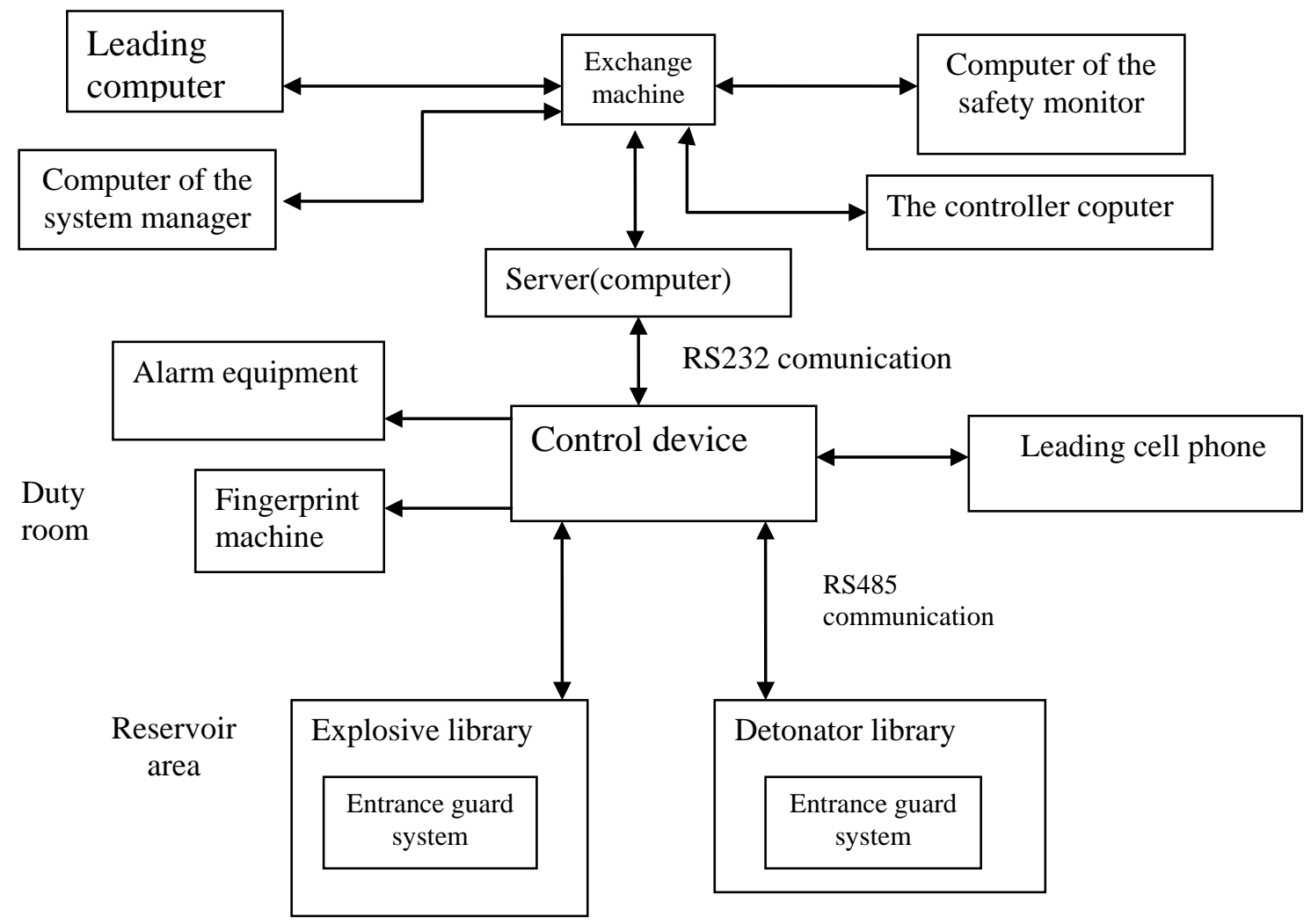

Fig.1. Intelligent management system architecture diagram of explosive magazine 


\section{Graphic description}

(1) The two box area of the lower part of the diagram are the area of the blasting equipment area, and the other area is the office area.

(2) Communication lines between the equipment in the reservoir area with the construction, the black line in the picture is a communication line, and the arrow represents the direction of data transmission

(3) The communication line between the reservoir area and the office area separate laying.

(4) The communication line in the reservoir area is twisted-pair, and if the distance between the reservoir and the office area is far away, it is difficult to lay,the fiber optic cable needed to use.

(5) Management terminal software of explosive devices needed to be installed in All PC, and the software of the terminal software can be assigned different operating rights according to different job responsibilities.

The monitoring and access management of explosive magazine and shot house in the reservoir area are controlled by the lower computer.The lower computer has the function of temperature and humidity, vibration response, time out alarm, fingerprint identification, electromagnetic lock control, data transmission and other functions, and it is controlled by single chip microcomputer[2]. System structure as shown in Fig. 2.

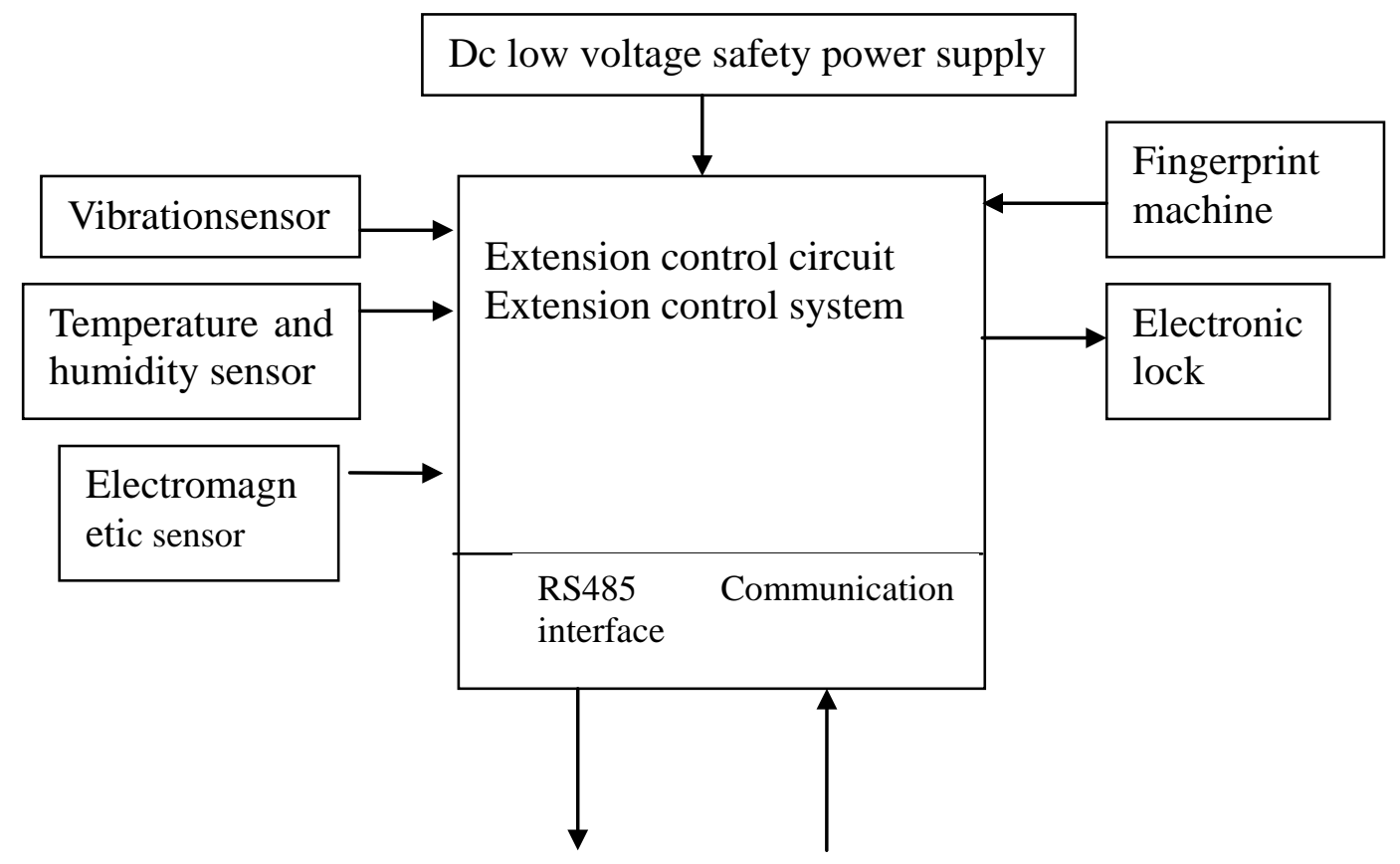

Fig.2. Lower computer system structure

In the duty room, there have a control device with stable performance. Its main function is to receive data from the lower computer and to send a lock command to the lower computer. The controller which use single-chip microcomputer technology can improve the reliability and security of the whole system. The main functions are fingerprint identification, information processing ability, data receiving and sending, alarm function, GSM communication, PC communication, human-computer interaction, etc. The function of network management and control can be achieved through server and server connectionin in the duty room. The function of single computer management and control can be achieved by a single computer and the controller connection, including the development and research of communication protocol, the design of authorization mode, the real time record of environmental parameters, and the development of the function of the record of explosive devices, the alarm record and the system fault self diagnosis. PC system to adopt C/S architecture, friendly interface, easy to control, the main function has a record of warehouse management, user management, temperature and humidity real-time, library door status real-time monitoring, inventory management, rights management, line condition monitoring, log 
management, query statistics, report forms printing, system configuration parameters, etc[3] .

\section{TCP/IP module interface circuit}

Circuit structure as shown in fig. 3

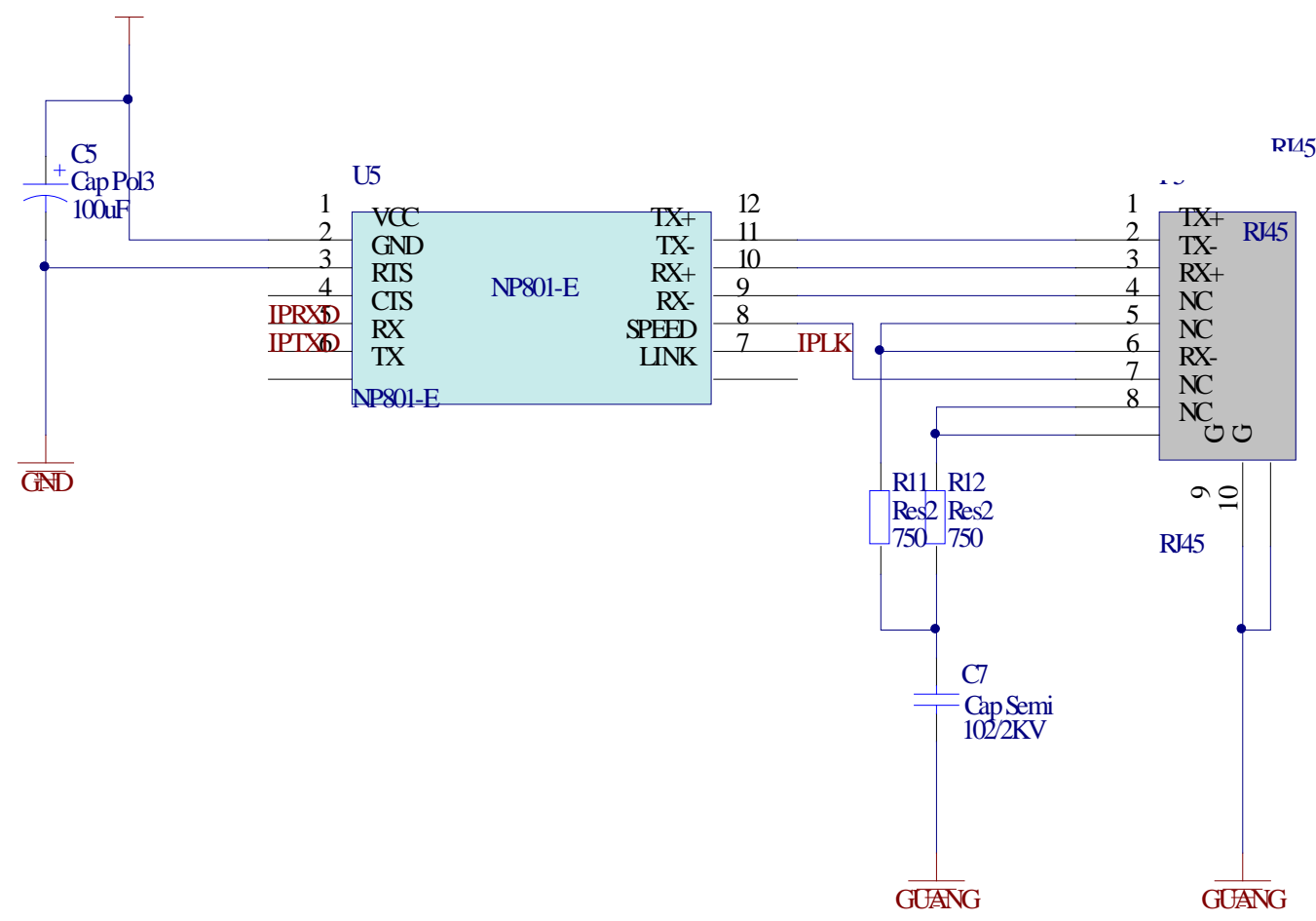

Fig. 3 TCP/IP module interface circuit

Pin elucidation:

TTL serial of 6pins

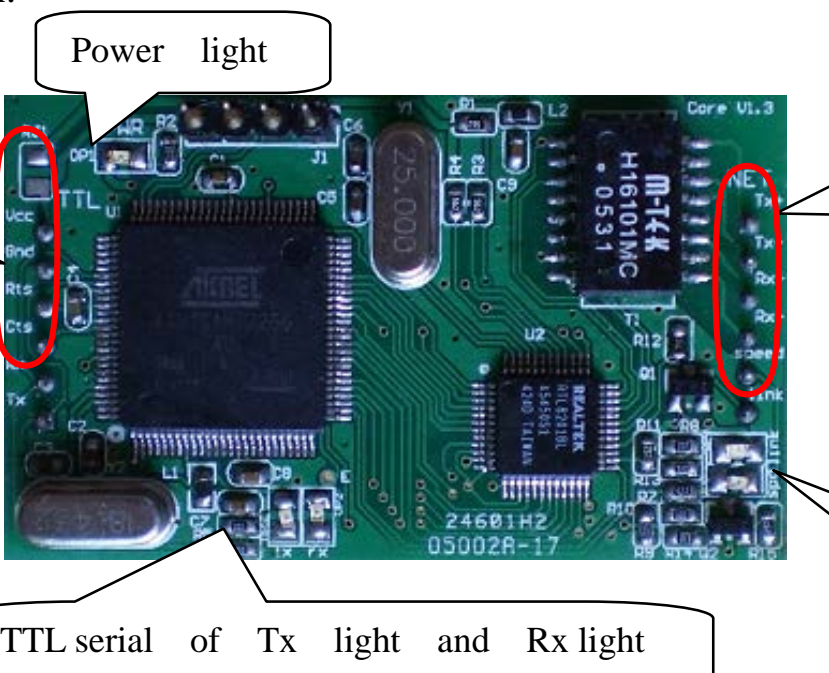

NET of Link light

And Speed light 


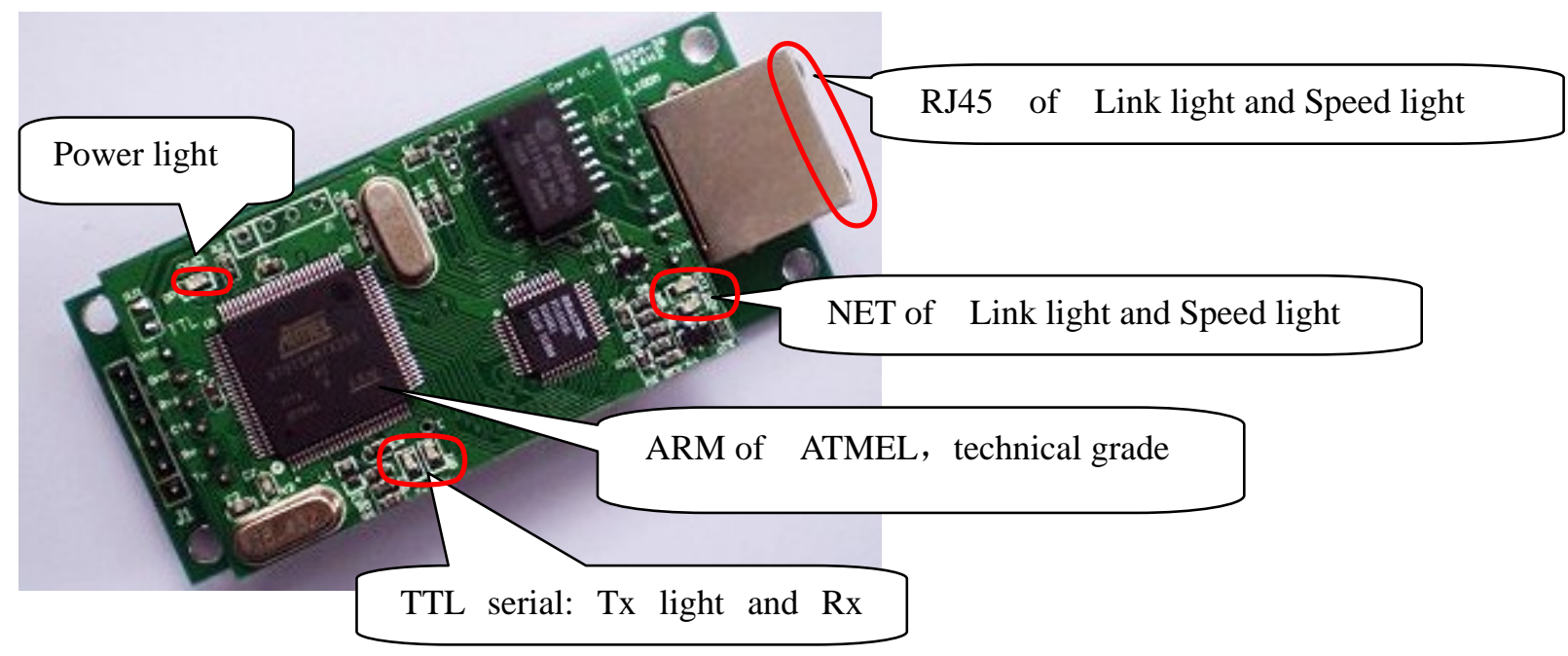

Indicator light can display data transmission direction, very convenient debugging.

Technical parameters

CPU : 32bits ARM; RAM: 64KB; Flash: 256KB; LAN: 100M/10Mbps, self-adaption, 1.5kV isolation protection conform to IEEE802.3 10BASE-T and 802.3u 100BASE-TX standards, Support the ARP, IP, ICMP, TCP, UDP and TCP/IP protocol.

Serial communication parameters: baud rate, Support 300bps to $115200 \mathrm{bps}$,data bit:5,6,7,8;examine: Even, Odd, Mark, Space, None; stop bit:1,1.5,2;flow control: CTS/RTS, XON/XOFF, None

Transmission distance: RS485/422 connector 1200m; RS232 connector 15m

The problems of library management system research are functions complexity and technical advances.

1). Data transfer protocol between the upper and lower computer is needed. System protocol for the study of management system to achieve more than 60 .

2). Data transmission format, the system of research and development are all adopt ASCII code for data transmission.

3). Read data from various forming products, such as the data read from the fingerprint machine, the data will be read out in various sensors.

4). UDP connection mode was adopted between upper and lower computer, the data transmission is higher, and the power consumption of the lower machine is reduced. User data protocol is a simple protocol for the transport layer for the data, UDP does not provide reliability, which send out the data that application program sent to IP layer, but can not guarantee that they can reach the destination. Because UDP does not establish a connection between the client and the server in the transmission data reported before, and no overtime retransmission mechanism, transmission speed is very fast.

\section{Establishment and application of equipment warehouse}

1).Build a new warehouse for initialization configuration, the mouse click warehouse management, then click 新建仓库 button, the new warehouse dialog will popup as follow.

After above information is completed, click the mouse. The information that new warehouse success will be prompted, the new warehouse successfully. 


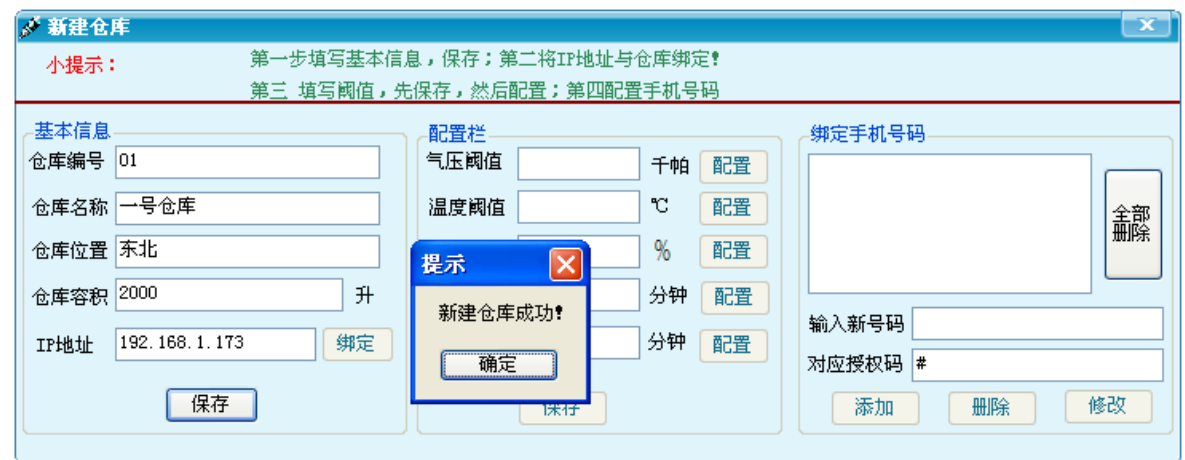

2). IP address and warehouse number binding

In the new warehouse interface, the mouse click the binding button. After a short time, it will popup the reminder warehouse number binding success, it indicates that the binding information was sent down to the warehouse control system[5]. At this point, click OK, shown as the following figure:

\begin{tabular}{|c|c|c|c|c|c|c|}
\hline \multicolumn{7}{|l|}{ 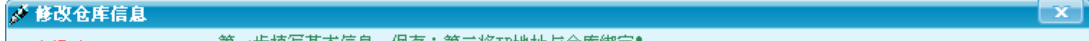 } \\
\hline 小提示： & \multicolumn{6}{|c|}{$\begin{array}{l}\text { 第一步埴写基本信息，保存；第二将IP地址与仓库绑定！ } \\
\text { 第三 埴写国值，先保存，然后配置；第四配置手机号码 } \\
\end{array}$} \\
\hline \multicolumn{7}{|l|}{ 基本信息 } \\
\hline 仓库编号 01 & \multicolumn{4}{|l|}{ 解计! } & & \multirow{2}{*}{ 全部册除 } \\
\hline 仓库名称 一号仓库 & \multirow{2}{*}{\multicolumn{4}{|c|}{ 仓库编号绑定成功! }} & & \\
\hline 仓库位置 东北 & & & & & & 校对手机号 \\
\hline 仓库容积 2000 & \multicolumn{4}{|c|}{ 确定 } & & \\
\hline \multirow{2}{*}{ IP地址 $\frac{192.168 .1 .173}{1 \text { 保存 }}$} & 娜定 & 授权超时 5 & 分钟 配置 & 输入授权码 & $\#$ & \\
\hline & & & & 添加 & 册除 & 修改 \\
\hline
\end{tabular}

\section{Part of the program about system operation}

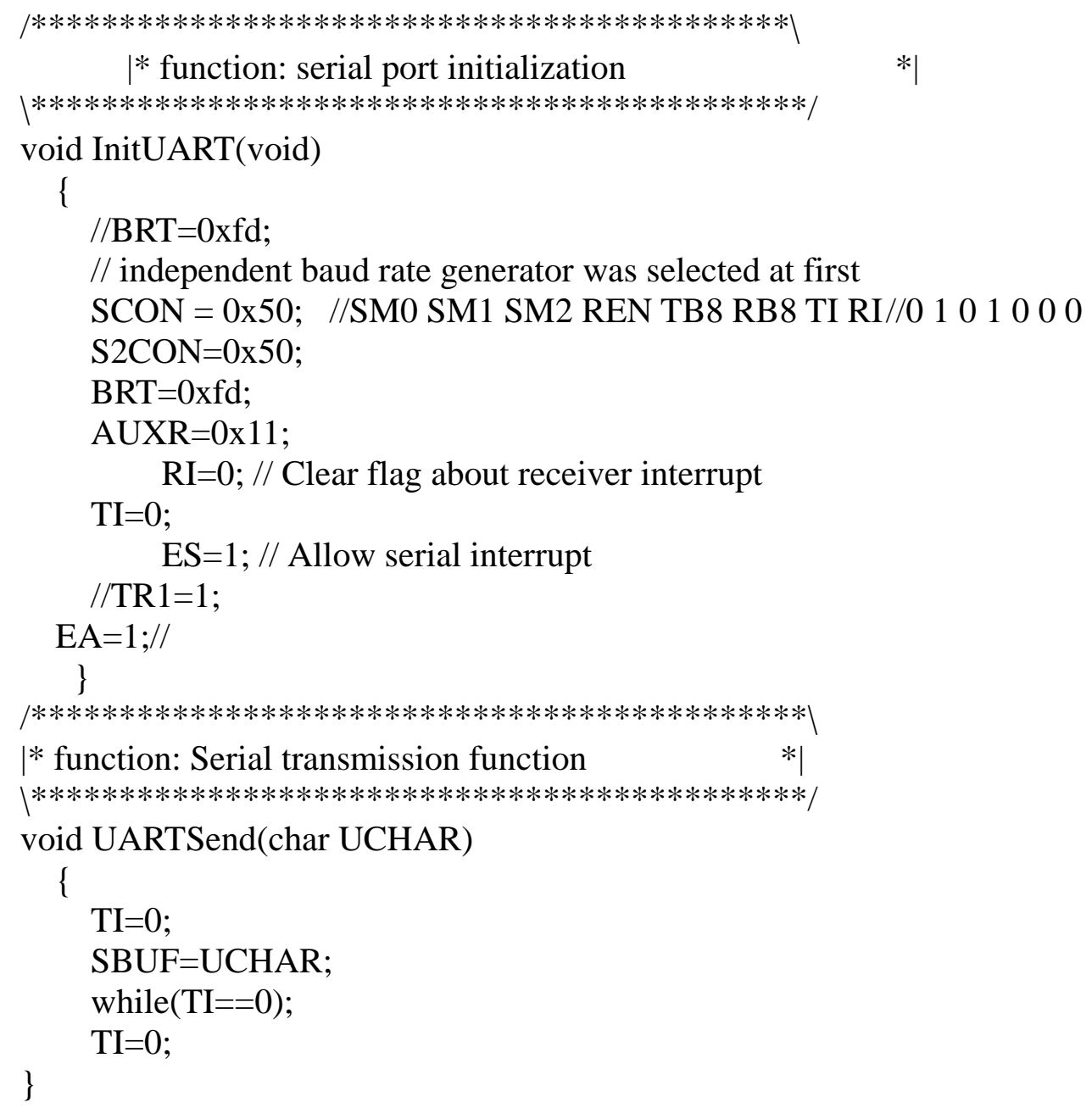

|* function: serial port initialization

$|* * * * * * * * * * * * * * * * * * * * * * * * * * * * * * * * * * * * * * * * * * * * *|$

void InitUART(void)

\{

//BRT=0xfd;

// independent baud rate generator was selected at first

SCON = 0x50; //SM0 SM1 SM2 REN TB8 RB8 TI RI//0 10101000

$\mathrm{S} 2 \mathrm{CON}=0 \times 50$;

$\mathrm{BRT}=0 \mathrm{xfd}$

AUXR=0x11;

$\mathrm{RI}=0$; / Clear flag about receiver interrupt

$\mathrm{TI}=0$;

$\mathrm{ES}=1 ;$ // Allow serial interrupt

//TR1=1;

$\mathrm{EA}=1 ; / /$

\}

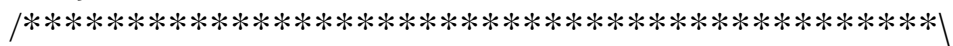

|* function: Serial transmission function

$|* * * * * * * * * * * * * * * * * * * * * * * * * * * * * * * * * * * * * * * * * * * *|$

void UARTSend(char UCHAR)

\{

$\mathrm{TI}=0$;

SBUF=UCHAR;

while(TI==0);

$\mathrm{TI}=0$; 


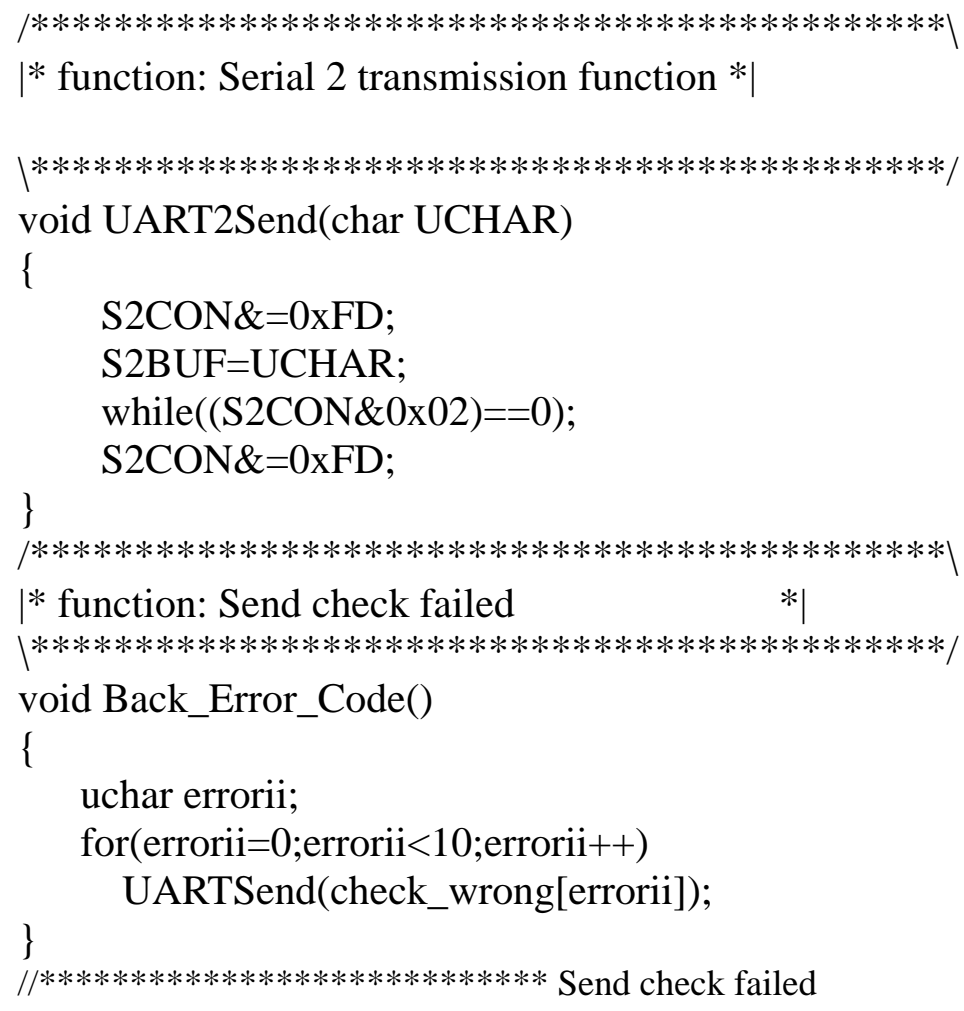

\section{Conclusion}

After experiment, the TCP/IP module interface circuit design of coal mine blasting equipment intelligent management system, intelligent control in the storage and use of the explosive devices is realized and stable operation, the expected parameters and the expected purpose can achieve, the design greatly improved the security and reliability of system.

\section{Acknowledgement}

In this paper, the research was sponsored by the Research Subject of Henan Province Education Technology and Equipment and Practice Education(GZS112) and by the Nature Science Foundation of Henan Province (Project No. 142300410286).

\section{Reference}

[1] CAI Chenwei.FAN Haijian.LIU Biyang. Design and Application of Gate Control System Based on Fingerprint Identification Technology [ J ] , Video Engineering. 2012.36(3):128-130

[2] Zhong XY,Wang BQ,Li B.Design of apparatus-cabinet controller based on EPM7128SLC84[J]. Journal Of Modern Electronics Technique.2006(1):110-112

[3] Zhao Ling.Design of alarm system based on photoelectric sensor[J].Electronic Test.2013,9(9):25-26

[4] Wang Min.Applications of Multi-serial Port Communication for MCS-51 Single Chip Computer[J].Electronic Test.2014,7:56-57

[5] Ran Fancan,Jiang Juping.Design and implementation of temperature acquisition system for supercomputer center[J].Cases of Application.2015,34(18):89-91 